Article

\title{
Intracellular Growth and Cell Cycle Progression are Dependent on (p)ppGpp Synthetase/Hydrolase in Brucella abortus
}

\author{
Mathilde Van der Henst, Elodie Carlier and Xavier De Bolle * \\ Unité de Recherche en Biologie des Microorganismes (URBM), University of Namur, \\ 61 rue de Bruxelles, 5000 Namur, Belgium; mathilde.vanderhenst@unamur.be (M.V.d.H.); \\ elodie.carlier@unamur.be (E.C.) \\ * Correspondence: xavier.debolle@unamur.be; Tel.: +32-81-72-44-38
}

Received: 12 June 2020; Accepted: 9 July 2020; Published: 14 July 2020

\begin{abstract}
Brucella abortus is a pathogenic bacterium able to proliferate inside host cells. During the first steps of its trafficking, it is able to block the progression of its cell cycle, remaining at the G1 stage for several hours, before it reaches its replication niche. We hypothesized that starvation mediated by guanosine tetra- or penta-phosphate, (p)ppGpp, could be involved in the cell cycle arrest. Rsh is the (p)ppGpp synthetase/hydrolase. A B. abortus $\Delta$ rsh mutant is unable to grow in minimal medium, it is unable to survive in stationary phase in rich medium and it is unable to proliferate inside RAW 264.7 macrophages. A strain producing the heterologous constitutive (p)ppGpp hydrolase Mesh1b is also unable to proliferate inside these macrophages. Altogether, these data suggest that (p)ppGpp is necessary to allow $B$. abortus to adapt to its intracellular growth conditions. The deletion of $d k s A$, proposed to mediate a part of the effect of (p)ppGpp on transcription, does not affect B. abortus growth in culture or inside macrophages. Expression of a gene coding for a constitutively active (p)ppGpp synthetase slows down growth in rich medium and inside macrophages. Using an mCherry-ParB fusion able to bind to the replication origin of the main chromosome of $B$. abortus, we observed that expression of the constitutive (p)ppGpp synthetase gene generates an accumulation of bacteria at the G1 phase. We thus propose that (p)ppGpp accumulation could be one of the factors contributing to the G1 arrest observed for B. abortus in RAW 264.7 macrophages.
\end{abstract}

Keywords: Brucella; cell cycle; (p)ppGpp; rsh

\section{Introduction}

Bacteria from the Brucella genus are the causative agents of brucellosis, a neglected disease which constitutes a worldwide anthropozoonosis. Brucella spp. are Gram negative alphaproteobacteria belonging to the Rhizobiales order [1]. Brucella abortus causes severe symptoms in mammals such as abortion in pregnant females and sterility in males. In humans, the disease is characterized by an undulant fever, also named Malta fever, and in the long term the infection leads to chronicity and symptoms such as arthritis, endocarditis and can have a fatal outcome without treatment [1]. In their hosts, Brucellae invade, survive and replicate inside professional and non-professional phagocytic cells such as macrophages and trophoblasts. Inside host cells, Brucellae are found in vacuoles named Brucella containing vacuoles (BCVs). In the first part of their trafficking, they successively harbor markers of early and late endosomes, a phase of the trafficking in which the bacterium does not proliferate $[2,3]$. This compartment presents a $\mathrm{pH}$ of about 4.0 to 4.5 and this acidification is essential for the successful establishment of B. suis infection [4]. Afterwards, the bacteria are found in BCVs having markers of the endoplasmic reticulum, where they replicate [5]. Later in the cellular infection, bacteria are found in vacuoles characterized by autophagy-related proteins [6]. 
Different cellular models for in vitro study of B. abortus infection of have been developed, such as the use of RAW 264.7 macrophages and HeLa epithelial cells. Some years ago, the investigations of the B. abortus infection process in these models revealed that the cell cycle regulation of B. abortus is linked to its virulence [2]. Indeed, for bacteria that did not segregate duplicated replication origins, the so-called G1 cells are more infectious than the S or G2 phase bacteria, i.e., bacteria currently replicating their genome or at the stage between the completion of genome replication genome and cell vision, respectively. More importantly, after internalization, bacteria remain in the G1 stage for up to $8 \mathrm{~h}$, depending the host cell type, in Lamp1-positive compartments before reaching the endoplasmic reticulum where $B$. abortus can restart its cell cycle, its DNA replication and actively proliferate [2]. During the first hours of the infection, in BCVs with endocytic markers, B. abortus encounters harsh conditions such as acidic stress [7] and alkylating stress [8]. In addition, it was already proposed that $B$. abortus has to face a starvation conditions inside host cells [9]. Starvation is the most obvious condition that could explain why B. abortus is blocked at the G1 stage of the cell cycle during the first phase of its intracellular trafficking in HeLa cells and RAW 264.7 macrophages. Starvation sensing is classically involving the synthesis of (p)ppGpp (guanosine penta- or tetra-phosphate), also called alarmone. The synthesis and degradation of (p)ppGpp are catalyzed by enzymes of the RelA/SpoT family, also called Rsh enzymes. It was found that $r$ sh mutants, which should be not able to produce (p)ppGpp anymore, are strongly impaired during in vitro infection as well as during murine infection $[10,11]$.

The alarmone (p)ppGpp is widely used by bacteria to quickly adapt to stress conditions such as nutrient starvation. The production and accumulation of this alarmone induces pleiotropic effects, modulating transcription and translation, that commonly result in cell cycle and DNA replication delay [12-15]. The ability to produce (p)ppGpp has been associated with virulence in bacterial pathogens belonging to relatively distant phylogenetic groups, such as Legionella pneumophila [16], Vibrio cholerae [17], and Mycobacterium tuberculosis [18]. In Escherichia coli, during the stringent response induced by starvation, (p)ppGpp binds directly to a site located at the interface between the $\beta^{\prime}$ and $\omega$ subunits of the RNA polymerase [19]. A second distinct site between the $\beta^{\prime}$ subunit and the DksA transcription factor has been shown to be bound by (p)ppGpp as well [20]. This interaction has been shown to enhance the transcriptional effects of DksA on the RNA polymerase, suggesting synergistic effects of DksA and (p)ppGpp together [20].

The RelA/SpoT homolog proteins are responsible for (p)ppGpp homeostasis. In E. coli, there are two enzymes of the Rsh family, RelA and SpoT [21]. SpoT contains a synthetase domain, a hydrolase domain, and two C-terminal regulatory domains; thus, this enzyme can both catalyze the production and the degradation of (p)ppGpp, respectively. RelA contains similar domains, however the functionality of the hydrolase domain of RelA has been lost during evolution, leading to a monofunctional enzyme that can only synthesize the alarmone [22]. In most alphaproteobacteria, including B. abortus, the production and the degradation of (p)ppGpp depends on one enzyme named Rsh (for RelA SpoT homolog) $[10,21]$.

In the present study, we analyzed the impact of alterations in (p)ppGpp synthesis or degradation on the growth, the cell cycle and the infection process of B. abortus. We show that mutants either unable to produce (p)ppGpp or producing a (p)ppGpp hydrolase are impaired for the infection process. In addition, our results show that expression of a constitutive (p)ppGpp synthetase negatively impacts growth and DNA replication of $B$. abortus, and also leads to a strong proliferation defect during infection of RAW 264.7 macrophages. We also observed that a B. abortus $d k s A$ null mutant was able to proliferate inside host cells as the wild type (WT) strain, suggesting that DksA is not crucially involved in the (p)ppGpp-dependent phenotypes observed during infection. These results suggest that adjustment of (p)ppGpp levels are crucial for the infection process in B. abortus. 


\section{Results}

\section{1. rsh Deletion Drastically Impacts Growth in Minimal Medium and the Infection Process}

We generated a $\Delta r s h$ strain by allelic replacement in B. abortus 544 and we assayed the growth of this strain in rich culture medium (2YT) as well as in Plommet minimal medium [23] supplemented with erythritol as a carbon source. The growth of $\Delta r s h$ in 2YT was similar to the WT strain during the exponential phase, but the shift into the stationary phase occurred later and at a higher optical density (OD) compared to the wild type strain (Figure 1A). To evaluate bacterial viability, we counted the colony forming units (CFUs) throughout the culture in liquid rich medium (Figure 2). The $\Delta r s h$ strain showed a clear survival defect during the stationary phase, marked by a decrease in CFUs between $24 \mathrm{~h}$ and $48 \mathrm{~h}$ compared to the WT and the complemented strain.

Because it has been shown in other bacteria that the stringent response is linked to nutrient availability, we tested the growth of the $\Delta r s h$ strain in Plommet minimal medium, supplemented with erythritol as a carbon source, to mimic starvation conditions. The $\Delta r s h$ showed a clear growth defect compared to the WT as the OD rapidly decreased during the mid-exponential phase, indicating that $\Delta r s h$ cannot grow and survive in this medium, as expected for a mutant unable to produce (p)ppGpp (Figure 1B).

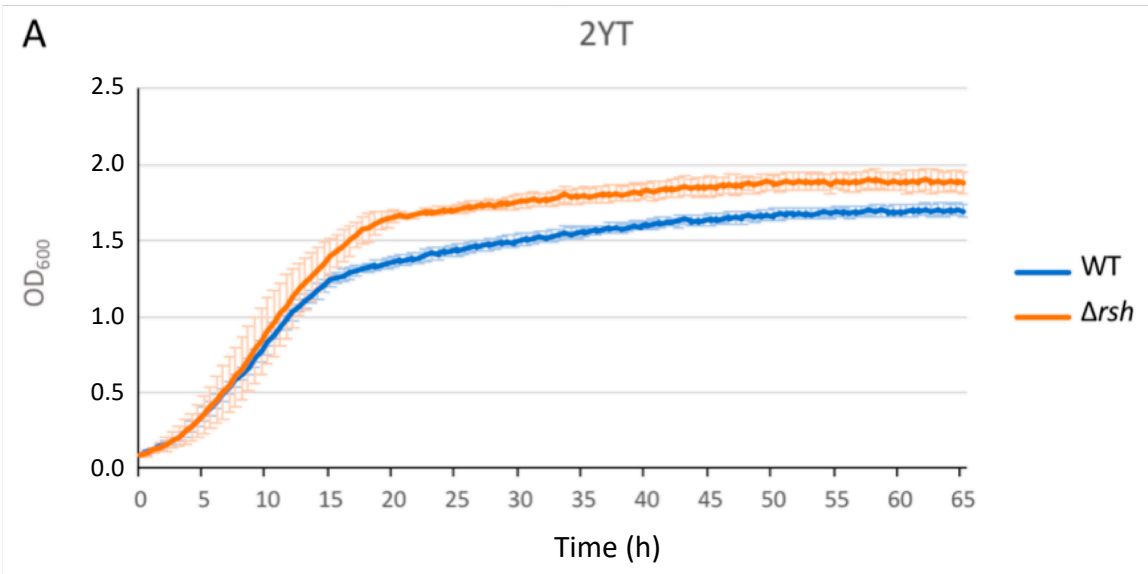

B

Plommet erythritol

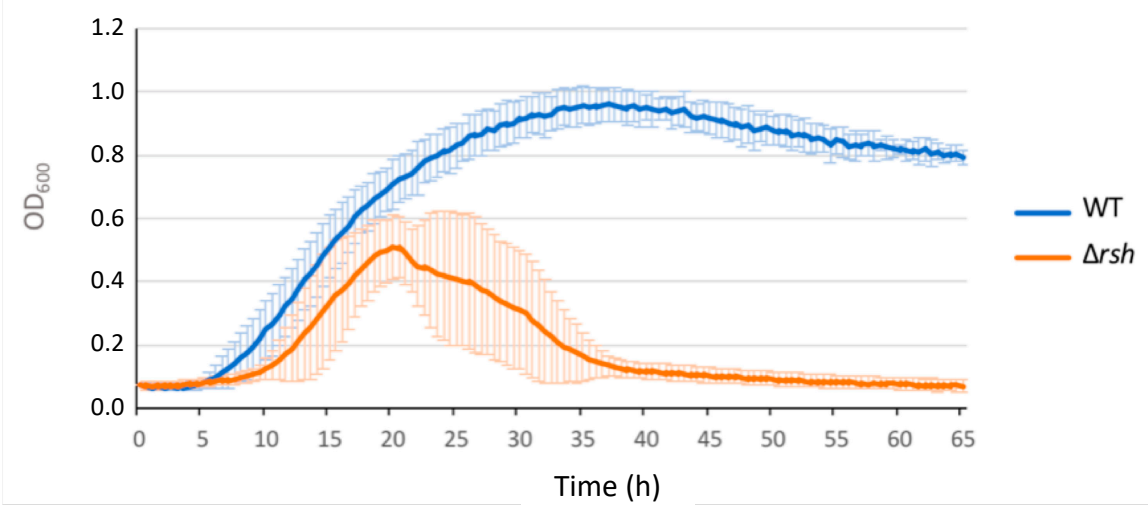

Figure 1. Growth of the $\Delta r s h$ mutant in 2YT rich medium (A) and in Plommet erythritol minimal medium (B). Strains were grown in liquid culture overnight in order to reach exponential phase. Cultures were then diluted at an optical density (OD) of 0.1 in 2YT medium. The OD of each strain was measured every $30 \mathrm{~min}$. The graph represents the means of a biological quadruplicate. The error bars represent the standard deviation for each time point. WT: wild type. 


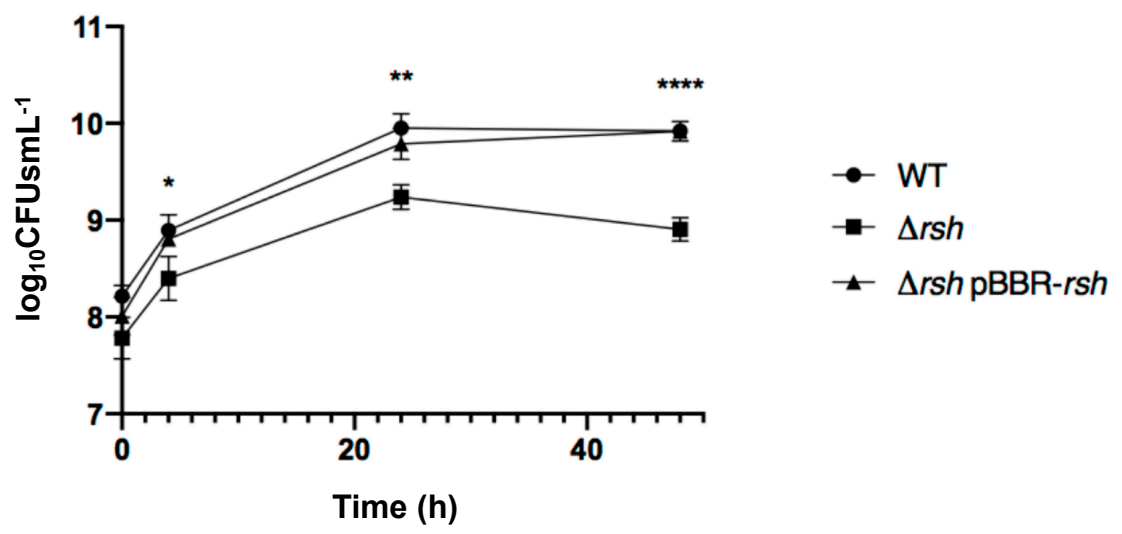

Figure 2. Survival and growth of B. abortus WT, $\Delta r s h$ and $\Delta r s h$ pBBR-rsh in 2YT rich medium. Strains were grown in liquid culture overnight in order to reach exponential phase. Cultures were then diluted at an OD of $0.1\left(3 \times 10^{8}\right.$ bacteria/mL for the WT strain) in 2YT medium. The numbers of live bacteria $\left(\log _{10}\right.$ CFUs $\left.\mathrm{mL}^{-1}\right)$ were determined at $0 \mathrm{~h}, 4 \mathrm{~h}, 24 \mathrm{~h}$ and $48 \mathrm{~h}$ by plating serial dilutions. Values represent the means of three independent experiments and the error bars represent the standard deviation. The asterisks mean significant for $p<0.05\left(^{*}\right) p<0.01\left(^{* *}\right) ; p<0.0001\left(^{* * *}\right)$, and the $p$ values were calculated by one-way ANOVA.

We tested the ability of $\Delta r s h$ to infect and multiply inside RAW 264.7 macrophages compared to the WT strain and the complemented strain by performing CFU counting throughout the cellular infection (Figure 3). The $\Delta r s h$ mutant showed a significant decrease in CFUs at $24 \mathrm{~h}$ post-infection compared to the WT strain, suggesting that the $r s h$ gene is required for intracellular proliferation. A slight but significant difference was also observed at $2 \mathrm{~h}$ post-infection between the WT and complemented strain (Figure 3), probably highlighting a low toxicity of the vector or a rsh overexpression effect.

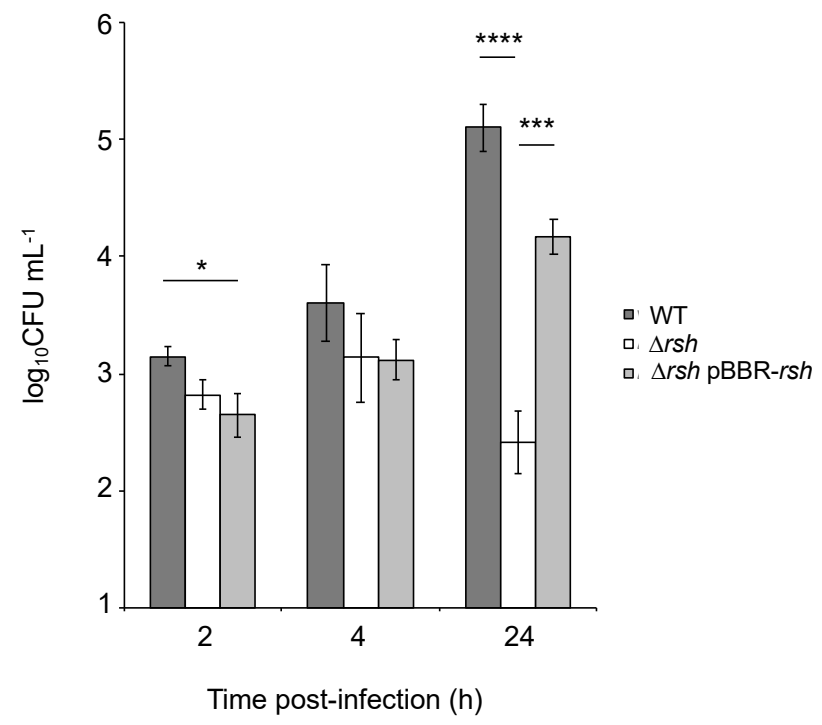

Figure 3. Survival and growth of B. abortus WT, $\Delta r s h$ and $\Delta r s h$ pBBR-rsh during infection of RAW 264.7 macrophages. Strains were grown in liquid culture overnight in order to reach exponential phase. Cultures were then diluted in Dulbecco's Modified Eagle's Medium (DMEM) to obtain a multiplicity of infection (MOI) of 50. The numbers of live bacteria $\left(\log _{10} \mathrm{CFUs} \mathrm{mL}^{-1}\right.$ of cellular lysate, $0.5 \mathrm{~mL}$ per well) were determined at $2 \mathrm{~h}, 4 \mathrm{~h}$, and $24 \mathrm{~h}$ by plating serial dilutions. Values represent the means of three independent experiments and the error bars represent the standard deviations. A one-way ANOVA test was performed as statistical analysis. The asterisks mean significant for $p<0.05\left(^{*}\right) ; p<0.001\left(^{* * *}\right)$; $p<0.0001\left({ }^{* * *}\right)$. 


\subsection{The Artificial Hydrolysis of (p)ppGpp Leads to a $\Delta r s h$ Phenotype during Infection}

Since Rsh is responsible for (p)ppGpp homeostasis and an $\Delta r s h$ mutant failed to proliferate inside RAW 264.7 cells, we tested the involvement of (p)ppGpp in the infection process. However, it is known that Rsh is involved in regulation networks through protein-protein contacts $[14,24]$ in other bacteria. Therefore, we cannot rule out that the absence of the Rsh protein, rather than the absence of (p)ppGpp, would be responsible for the defect observed in infection. This is reinforced by the observation that mutants for homologs of the glutamine-dependent control pathway of Rsh are also attenuated in RAW 264.7 macrophages [25]. We thus generated a strain in which (p)ppGpp is hydrolyzed by a strong (p)ppGpp hydrolase, a product of the mesh1 gene from Drosophila melanogaster [26]. Indeed, it was shown that Mesh1 was active in vitro and in vivo [26]. We thus expect this heterologous enzyme to be constitutive in $B$. abortus. We adapted the mesh 1 coding sequence for the codon bias of B. abortus and expressed the resulting coding sequence on a medium copy replicative plasmid, leading to the B. abortus pBBRi-mesh $1 b$ strain. Interestingly, this strain showed a clear decrease in CFUs at $24 \mathrm{~h}$ post-infection of RAW 264.7 macrophages (Figure 4), which is consistent with a crucial role played by (p)ppGpp to allow growth inside host cells, as suggested above.

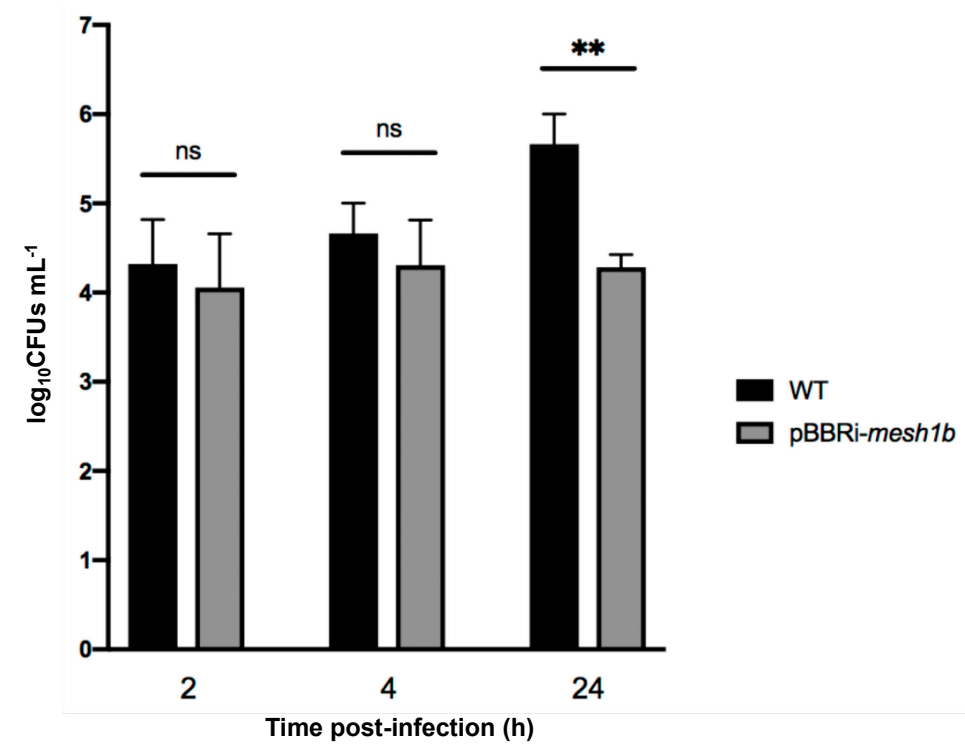

Figure 4. Survival and growth of B. abortus WT and pBBRi-mesh $1 b$ during infection of RAW 264.7 macrophages. Strains were grown in liquid culture overnight in order to reach exponential phase. Cultures were then diluted in DMEM to obtain a MOI of 50 . The numbers of live bacteria $\left(\log _{10} \mathrm{CFUs}\right.$ $\mathrm{mL}^{-1}$ ) were determined at $2 \mathrm{~h}, 4 \mathrm{~h}$, and $24 \mathrm{~h}$ post-infection by plating serial dilutions. Values represent the means of three independent experiments and the error bars represent the standard deviation. A Student $t$ test was performed for the comparison of the two strains. The asterisks mean significant for $p<0.01\left(^{* *}\right)$ and "ns" means "not significant".

\subsection{Expression of a Constitutive Allele for a (p)ppGpp Synthetase Impacts Bacterial Growth and Chromosome Replication}

In order to get more insight about the role of (p)ppGpp in B. abortus, we constructed a strain that artificially produces this alarmone. We used a truncated version of the relA gene from $E$. coli, relA' [12] that removes the $\mathrm{C}$-terminal regulatory domains of the encoded protein. The rel $A^{\prime}$ coding sequence was inserted downstream of an isopropyl $\beta$-D-1-thiogalactoside (IPTG)-inducible promoter on the pSRK replicative plasmid [27]. The resulting strain, named $p S R K-r e l A^{\prime}$, is supposed to produce (p)ppGpp synthetase when IPTG is added to the medium. As a negative control, we used the $p S R K$-relA $A^{\prime *}$ strain containing the point mutation $\mathrm{E} 335 \mathrm{Q}$, which leads to a catalytically dead protein. Since the detection of (p)ppGpp levels using ${ }^{32} \mathrm{P}$ is not compatible with our biosafety level 3 set up, we tried to gain indirect 
evidence that (p)ppGpp is indeed produced when the expression of $r e l A^{\prime}$ is induced. We assayed the growth of the $p S R K-r e l A^{\prime}$ and $p S R K$-rel $A^{\prime *}$ strains in rich culture medium with or without IPTG induction. The $p S R K-r e l A^{\prime}, p S R K-r e l A^{\prime *}$ and WT strains grew equally in 2YT; however, when IPTG was added to the medium, a growth delay was only observed for the $p S R K$-relA' strain (Figure 5). This observation is consistent with the production of (p)ppGpp levels that are sufficient to limit growth when relA' expression is induced.

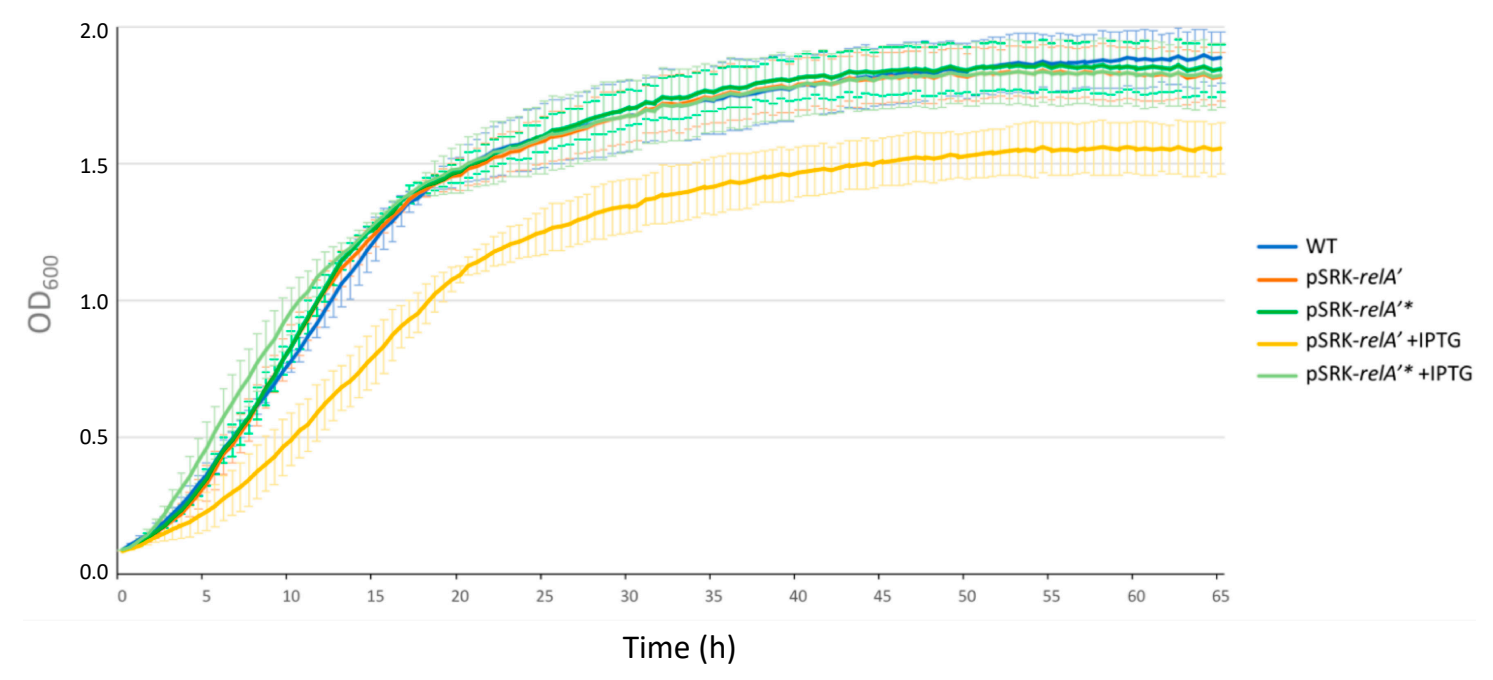

Figure 5. Growth curve in rich culture medium for the WT, $p S R K$-relA' and $p S R K$-relA** with or without IPTG. Strains were grown in liquid culture (2YT medium) overnight in order to reach exponential phase. Cultures were then diluted at an OD of 0.1 in 2YT medium supplemented or not with IPTG. The OD of each strain was measured every $30 \mathrm{~min}$. The graph represents the means of a biological triplicate. The error bars represent the standard deviation for 3 biological replicates for each time point.

Since it was already reported that (p)ppGpp has an impact on DNA replication in C. crescentus and E. coli [12-15], we took advantage of a B. abortus strain allowing us to monitor the chromosomal replication status at the single cell level in order to study the impact of (p)ppGpp overproduction on DNA replication. This strain expresses an $m$ Cherry-parB allele that allows us to highlight the segregation of replication origin(s) of chromosome I. In this strain, one mCherry focus means that segregation has not yet started and the bacterium is probably in the G1 phase of the cell cycle, and two mCherry foci correspond to two segregated replication origins, meaning that the bacterium has already started replication and is thus in the S or G2 phase of the cell cycle [2]. The $p S R K-r e l A^{\prime}$ and $p S R K$-relA $A^{\prime *}$ plasmids were inserted in a B. abortus $m$ Cherry-parB strain and we counted the number of G1 bacteria every two hours for $6 \mathrm{~h}$ after the inoculation of bacteria in rich medium, with or without IPTG. Interestingly, we observed an increase in the proportion of G1 bacteria over the time of induction with IPTG for the $p S R K$-relA' strain (Supplementary Figure S3). The proportion of G1 bacteria of the non-induced $p S R K-r e l A^{\prime}$ and both the induced or non-induced $p S R K-r e l A^{\prime *}$ remained stable after the addition of IPTG (Figure 6). These results strongly suggested that artificial induction of (p)ppGpp synthesis could delay the transition between the G1 phase to the $S$ phase and subsequently have an impact on the initiation of chromosomal replication in B. abortus. 
A

oril
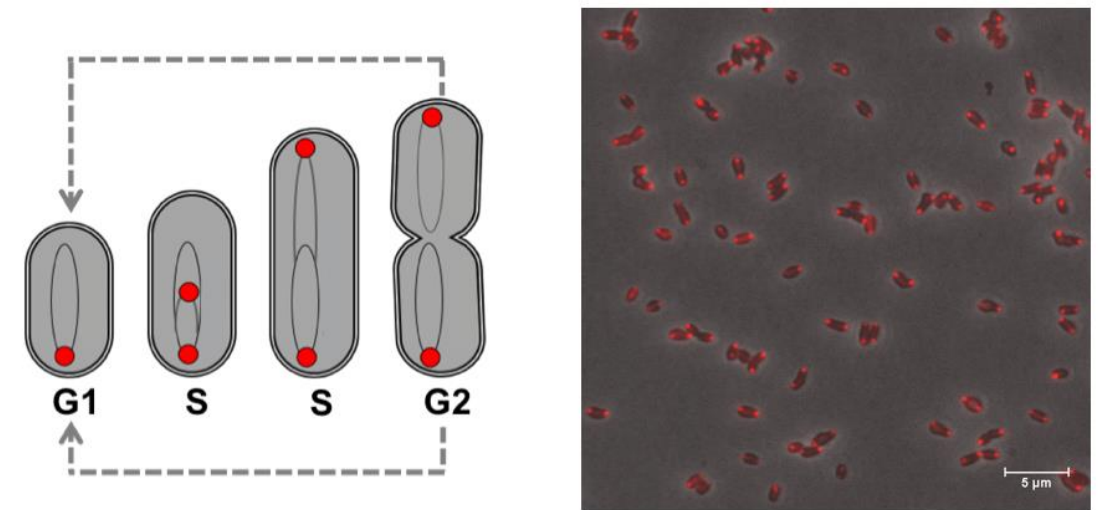

B

oril

C

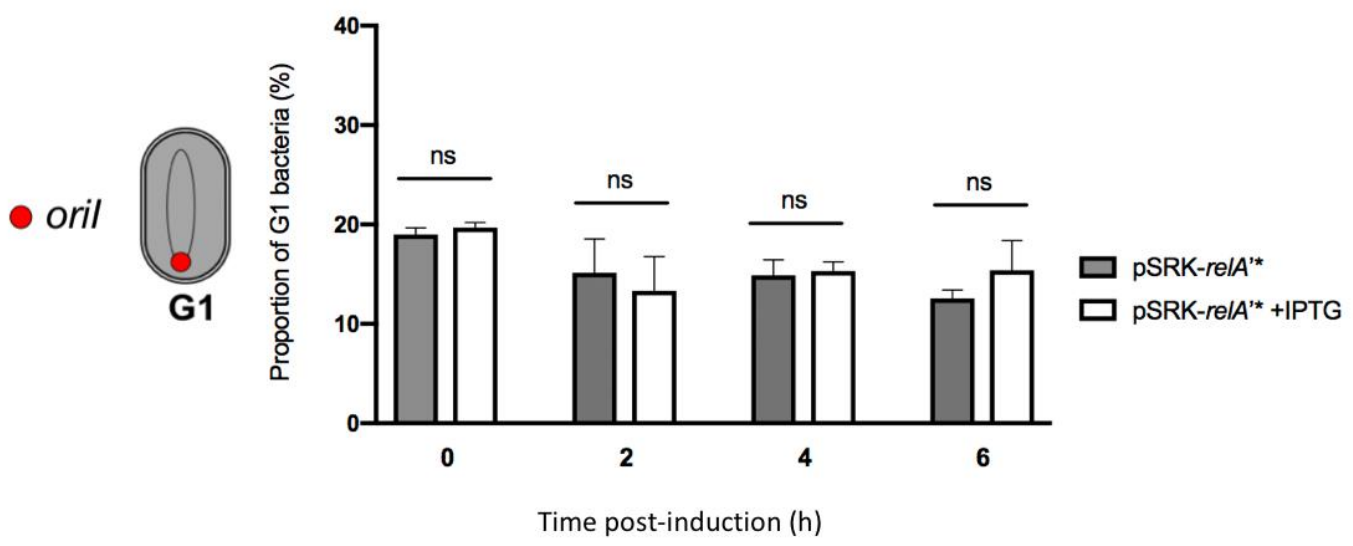

Figure 6. Proportion of G1 bacteria in rich culture medium with or without IPTG for the $p S R K$-rel $A^{\prime}$ and $p S R K$-relA $A^{* *}$ strains. (A) Schematic drawing of the mCherry-ParB localization throughout the cell cycle [2] and fluorescence microscopy of the $p S R K$-relA' $m$ Cherry-parB strain. Scale bar represents $5 \mu \mathrm{m}$. (B) Strains were grown in liquid culture (2YT medium) overnight in order to reach exponential phase. Cultures were then diluted to an OD of 0.1 in 2YT medium supplemented or not with IPTG. Samples were taken every $2 \mathrm{~h}$, placed on a phosphate-buffered saline (PBS) agarose pad and observed with a fluorescence microscope. Bacteria in G1 phase (presenting only one focus of mCherry-ParB) were counted for each time post-induction. Error bars represent the standard deviation from the means of three independent experiments (biological triplicates). The significant differences are indicated by $p<0.05\left(^{*}\right), p<0.01\left(^{* *}\right)$ and $p<0.001\left(^{* * *}\right)$; "ns" means not significant. The number of bacteria considered in these triplicate experiments are detailed in Supplementary Table S1. 


\subsection{Induced Production of a Constitutive (p)ppGpp Synthetase Leads to a Proliferation Defect during Infection}

Since (p)ppGpp overproduction seemed to have an impact on replication, i.e., an increase of the proportion of G1 cells in the bacterial population, and that the G1 bacteria are more infectious, we decided to investigate the effect of overproduction of (p)ppGpp on the infection process. We infected RAW 264.7 macrophages with the $p S R K$-relA' strain induced or not with IPTG (Figure 7). The IPTG was kept in the cell culture medium during the infection for the induced condition. We first observed that bacterial internalization is not enhanced by the increase in the proportion of bacteria in the G1 phase of the cell cycle. We also observed that induction of $p S R K$-rel $A^{\prime}$ induced a strong defect in intracellular proliferation compared to the WT and uninduced $p S R K-r e l A^{\prime}$ conditions. This result suggests that overproduction of (p)ppGpp during infection prevents growth in the intracellular niche.

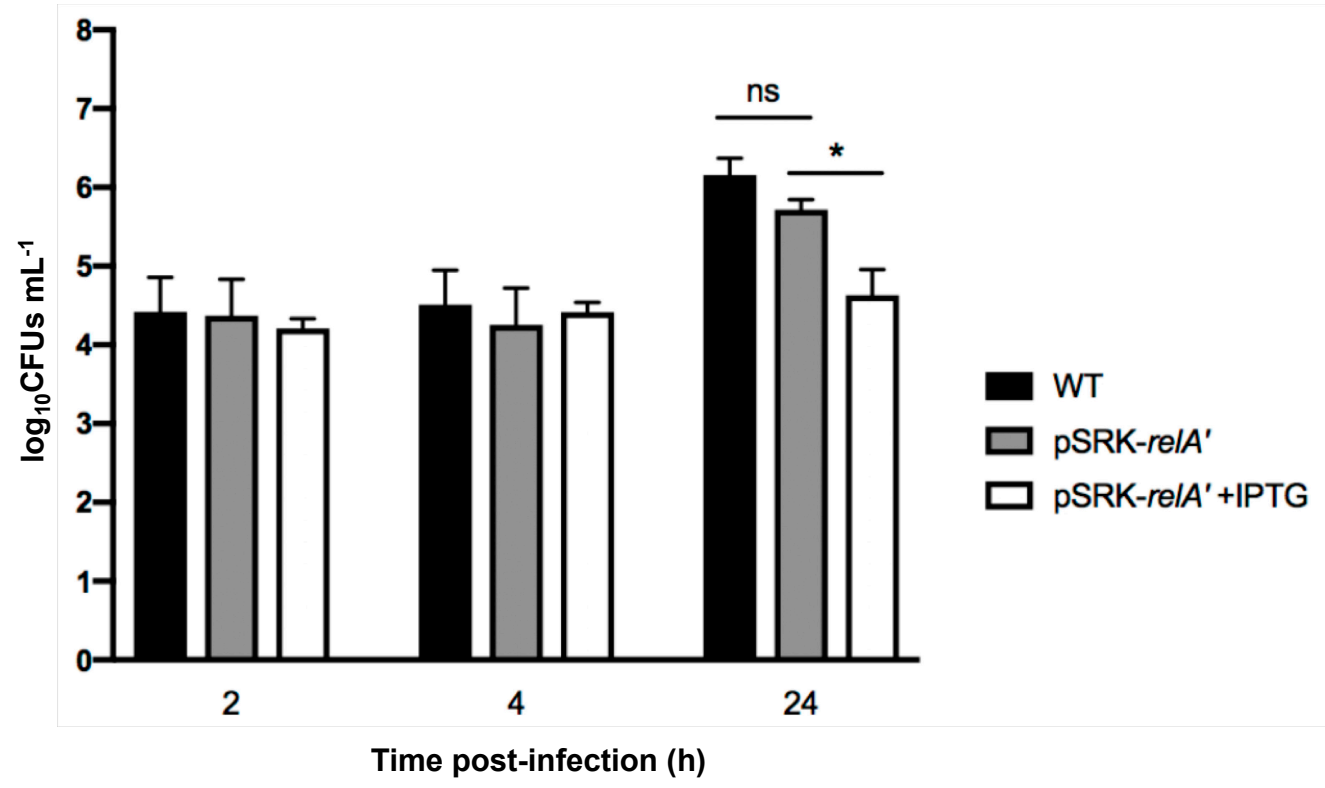

Figure 7. Survival of $B$. abortus WT and $p S R K$-relA' strains with and without IPTG during infection of RAW 264.7 macrophages. Strains were grown in liquid culture overnight in order to reach exponential phase. Cultures were then diluted at an OD of 0.1 with or without IPTG $(1 \mathrm{mM})$ and were incubated for $3 \mathrm{~h}$ at $37^{\circ} \mathrm{C}$. Cultures were then diluted in DMEM with or without IPTG $(10 \mathrm{mM})$ to obtain a MOI of 50. Concentrations of live bacteria $\left(\log _{10} \mathrm{CFUs} \mathrm{mL}{ }^{-1}\right)$ were determined at $0 \mathrm{~h}, 4 \mathrm{~h}$, and $24 \mathrm{~h}$ post-infection by plating serial dilutions. Values represent the means of three independent experiments and the error bars represent the standard deviation. A Student's $\mathrm{t}$ test was performed as statistical analysis. The asterisks mean significant for $p<0.05\left(^{*}\right)$ and "ns" means "not significant".

\subsection{Dks A Is Not Required during the Infection Process}

Because (p)ppGpp seemed important during host infection and DksA is involved in a part of the (p)ppGpp transcriptional response in other species, we tested the ability of $\Delta d k s A$ to infect and proliferate inside RAW 264.7 macrophages. No difference in CFUs was observed between WT and $\triangle d k s A$ strains (Supplementary Figure S1), meaning that DksA is not crucially involved in the infection process and that the phenotype observed for (p)ppGpp-deprived mutants ( $\triangle r s h$ and $p B B R i$-mesh $1 b)$ is probably not mediated by DksA.

\section{Discussion}

Brucella abortus is able to control its cell cycle progression when it is inside host cells, particularly the replication and segregation of its replication origins [2]. However, the molecular mechanisms involved in this control are unknown. Since convincing data show that the ability to adapt to starvation is a key factor for the success of cellular infections by Brucella melitensis and Brucella suis [10,11], 
we further investigate the role of the (p)ppGpp, the alarmone produced in the presence of starvation conditions, and the Rsh enzyme that is proposed to synthesize this alarmone. We first confirmed that in B. abortus 544, like in other Brucella strains, Rsh is crucial for the success of a cellular infection (Figure 3), for the survival in stationary phase (Figure 2) and the growth in minimal medium (Figure 1). In agreement with the absence of proliferation of the rsh mutant in macrophages, a strain constitutively producing a (p)ppGpp hydrolase (Mesh1b) from Drosophila melanogaster is also unable to grow in RAW 264.7 macrophages. We observed that the survival of $p B B R$-mesh $1 b$ strain is less severely impacted during infection than the $\Delta r s h$ strain. One could imagine that this intermediate phenotype is due to the presence of residual alarmone in the $p B B R$-mesh $1 b$ strain, while it is not the case in the $\Delta r s h$ strain since the (p)ppGpp synthetase domain is not present. Another explanation for these different phenotypes could be that Rsh plays additional role(s) for survival in infection than the regulation of (p)ppGpp homeostasis. Interestingly, a mutant overproducing (p)ppGpp is also unable to proliferate in these cells, suggesting that the (p)ppGpp level should be in a specific range of concentration to allow cellular infection; having too much or not enough (p)ppGpp would be detrimental for the success of the cellular infection.

What does (p)ppGpp control and how is Rsh regulated? It was shown that the absence of $r$ sh in B. suis affects the transcription of genes known to be involved in virulence [28], such as pyrB, which was shown to be essential for B. abortus proliferation in RAW 264.7 macrophages [25]. It is thus likely that a minimum level of (p)ppGpp would be required for the success of a cellular infection by B. abortus. It was shown that the glutamine pool modulates Rsh (called SpoT) in the model alpha-proteobacterium Caulobacter crescentus through the phosphotransferase system (PTS) [14]. Interestingly, mutants for components of this system were found to be attenuated in RAW 264.7 macrophages [25]. Moreover, PTS and the two-component regulator BvrR control the expression of the virB operon [29,30], coding for a type IV secretion system that is crucial for intracellular proliferation in most cell types [31]. These data indicate that a quite complex regulation network is probably linking Rsh control and virulence. However, the molecular mechanisms controlling Rsh activity in B. abortus are unknown and deserve further investigation.

One striking conclusion of our data is the moderate effect that (p)ppGpp overproduction has on the proportion of bacteria at the G1 stage of the cell cycle. Indeed, while overproduction seems to be sufficient to impair growth inside host cells (Figure 7), the proportion of G1 after $6 \mathrm{~h}$ of induction is about a third of the culture while it is approximately $15 \%$ in the absence of induction (Figure 6). Since the cell cycle takes about $3 \mathrm{~h}$ in the conditions tested, it is likely that only a fraction of the bacteria arrested their cell cycle at the G1 stage. Inside host cells, the proportion of G1 cells is about $75 \%$ and remains stable for 2 to $4 \mathrm{~h}$ at least [2], suggesting that other mechanisms are probably involved in the control of the cell cycle in host cells, early in the trafficking. These mechanisms could involve the acidic nature of the $\mathrm{BCV}$, or the diffusion sensing proposed to occur through a regulation system homologous to quorum sensing [32]. More investigations are thus needed to discover the multiple factors involved in the cell cycle control of B. abortus inside host cells.

\section{Materials and Methods}

\subsection{Strains and Growth Conditions}

The reference strain B. abortus 544 was used for all experiments and was grown on solid or in liquid 2YT medium (LB $32 \mathrm{~g} / \mathrm{L}$ Invitrogen, Yeast Extract $5 \mathrm{~g} / \mathrm{L}, \mathrm{BD}$ and Peptone $6 \mathrm{~g} / \mathrm{L}, \mathrm{BD}$ ) at $37^{\circ} \mathrm{C}$. E. coli strain DH10B was used for plasmid constructs and the conjugative strain E. coli S17-1 was used for mating with B. abortus. Both strains were cultivated in LB medium (Luria Bertani, Casein Hydrolysate $10 \mathrm{~g} / \mathrm{L}, \mathrm{NaCl} 5 \mathrm{~g} / \mathrm{L}$, Yeast Extract $5 \mathrm{~g} / \mathrm{L}$ ) at $37^{\circ} \mathrm{C}$. Depending on the plasmid used, different selection antibiotics were added to the culture medium: ampicillin $(100 \mu \mathrm{g} / \mathrm{mL})$; carbenicillin $(100 \mu \mathrm{g} / \mathrm{mL})$; kanamycin $(50 \mu \mathrm{g} / \mathrm{mL}$ for the replicative plasmid, and $10 \mu \mathrm{g} / \mathrm{mL}$ for the integrated plasmid); nalidixic acid $(25 \mu \mathrm{L} / \mathrm{mL})$; chloramphenicol $(20 \mu \mathrm{g} / \mathrm{mL}$ for the replicative plasmid and $4 \mu \mathrm{g} / \mathrm{mL}$ for the integrated 
plasmid). Isopropyl $\beta$-d-1-thiogalactopyranoside (IPTG) was used at a concentration of $1 \mathrm{mM}$ in bacterial liquid culture and at $10 \mathrm{mM}$ in the mammalian cell culture medium during cellular infections. When the $\Delta r s h$ mutant was constructed, we added casamino acids 0.5\% (BactoTM Casamino Acids from Thermo Fisher, Waltham, MA, USA) in the conjugation medium.

\subsection{Strains Construction}

Deletion strains were constructed by allelic exchange using the pNPTS138 vectors (M. R. K. Alley, Imperial College of Science, London, UK) carrying the upstream and the downstream regions of the targeted gene. The primer sequences used for amplification of the upstream region of the $d k s A$ gene were $5^{\prime}$-ttGGATCCcaagcgccagatcttca-3' and 5'-ttGAATTCttcactcattctgaatcacccc-3'. The primer sequences used for amplification of the downstream region of the $d k s A$ gene were 5'-ttGAATTC tgatatcgaataatggtttggaaa-3' and 5'-ttAAGCTTcgcccagcttcaaattac-3'.

We used the rsh deletion plasmid pMQ203 (provided by M. Quebatte, Biozentrum, Basel), containing the upstream and downstream regions of $r s h$ amplified with the following hybridization sequences: $\quad 5^{\prime}$-ccggatgatctgaaggaa-3', 5'-gcgcatcatctgccgaaa-3' and $5^{\prime}$-gtctgggacctcaagcat-3', $5^{\prime}$-cccgtggtgacgatatct-3'.

The $\Delta r s h$ pBBR-rsh strain was generated by inserting the pBBR-rsh in the $\Delta r s h$ strain. The pBBR-rsh was constructed by cloning the endogenous promoter of $r s h$ and the $r s h$ coding sequence, amplified with the primers 5'-aaaCTCGAGgcgagattgccgatgaga-3' and 5'-aaaCTGCAGctatccgttcacacgctttg-3'.

The pBBRi-mesh $1 b$ strain was constructed by inserting the coding sequence mesh $1 b$ in the pBBRi plasmid. The sequence of mesh $1 b$ was adapted to the codon usage of Brucella, and is available in Supplementary Figure S2.

The $m$ Cherry-parB strains containing pSRK-rel $A^{\prime}$ and pSRK-relA $A^{\prime *}$ were created using the Tn7 system [33] which consists in transposition of mini-Tn7 expressing $m$ Cherry-parB under the control of the PgidA promoter as previously reported [2] and the resistance cassette to ampicillin/carbenicillin under the control of Pbla promoter at the glmS locus of B. abortus. The primer sequences used for the amplification of PgidA-mCherry-parB and Pbla-amp were 5'-cgcggatcctctgtggaatcctgtttgttg-3', 5'-AGCGGATACATATTTGAActagctttgaagacggcg-3' and 5'-TTCAAATATGTATCCGCTCATGA-3', 5'-cgggatccTTACCAATGCTTAATCAGTGAGG-3'.

\subsection{Growth Assays}

The bacterial growth curves were performed using a bioscreen (Epoch2 Microplate Photospectrometer from BioTek). Bacterial cultures in the exponential phase of growth were washed two times with PBS and were normalized at an OD of 0.1 in a given medium. A $200 \mu \mathrm{L}$ aliquot of the normalized culture was transferred to a plate and each condition was performed in technical triplicate $(3 \times 200 \mu \mathrm{L})$. The plate was incubated at $37^{\circ} \mathrm{C}$ with shaking and the $\mathrm{OD}_{600}$ of each well was measured every $30 \mathrm{~min}$. One biological replicate constitutes the mean of three technical replicates and experiments were repeated at least three times to obtain biological triplicates.

\subsection{Survival Assays}

Bacterial cultures in the exponential phase of growth were normalized at an OD of 0.1 in 2YT liquid medium. Serial dilutions were plated on 2YT solid medium at different time points and plates were incubated at $37^{\circ} \mathrm{C}$.

\subsection{Infections of RAW 264.7 Macrophages}

RAW macrophages were put in wells in DMEM medium (with decomplemented bovine serum, glucose, glutamine, and no pyruvate, Gibco $\left.{ }^{\circledR}\right)$ to have $1 \times 10^{5}$ cells $/ \mathrm{mL}$. B. abortus 544 was grown in $2 \mathrm{YT}$ at $37^{\circ} \mathrm{C}$ until exponential phase. The OD of the bacterial culture was measured, and dilutions were performed to have a MOI equal to 50 (50 times more bacteria than macrophages). An input control was performed for each condition by plating bacteria on a 2YT agar plate before infecting cells. 
Cell medium was removed to add the appropriate bacterial dilution. The mix was centrifuged for $10 \mathrm{~min}$ at $1200 \mathrm{rpm}\left(4{ }^{\circ} \mathrm{C}\right)$ and then incubated at $37^{\circ} \mathrm{C}$ with $5 \% \mathrm{CO}_{2}$ (this time point is set as time zero). After one hour of incubation, medium was removed and replaced by medium containing gentamycin $(50 \mu \mathrm{g} / \mathrm{mL})$ for $1 \mathrm{~h}$ in order to kill extracellular bacteria, and then by medium containing gentamycin $(10 \mu \mathrm{g} / \mathrm{mL})$. Note that for the experiments using IPTG, the IPTG $(10 \mathrm{mM})$ was kept during all the steps of the infection. At either $2 \mathrm{~h}, 4 \mathrm{~h}$ or $24 \mathrm{~h}$ post infection, cells were first washed with sterile PBS and were then incubated in PBS + Triton $0.1 \%$ at $37^{\circ} \mathrm{C}$ for $10 \mathrm{~min}$ in order to lyse the cells while keeping bacteria alive. After that, cells were flushed and lysates were harvested. Serial dilutions were performed and each dilution was spotted on $2 \mathrm{YT}$ agar plates and incubated at $37^{\circ} \mathrm{C}$.

\subsection{Infections of HeLa Cells}

HeLa cells were plated in wells in DMEM medium (with sodium pyruvate, non-essential amino acid, glucose, glutamine, and no pyruvate, Gibco ${ }^{\circledR}$ ) at $4 \times 10^{4}$ cells $/ \mathrm{mL}$. B. abortus 544 was grown in 2YT at $37^{\circ} \mathrm{C}$ until exponential phase, the OD of the bacterial culture was measured, and dilutions were performed to have a MOI equal to 300. An input control was performed for each condition by plating bacteria on a 2YT agar plate before infecting cells. Prior to infections in the presence of IPTG (see below), relA' expression was induced $3 \mathrm{~h}$ before infection with IPTG (1 $\mathrm{mM}$ in YT medium). Cell medium was removed to add the appropriate bacterial dilution. The mix was centrifuged for $10 \mathrm{~min}$ at $1200 \mathrm{rpm}\left(4^{\circ} \mathrm{C}\right)$ and incubated at $37^{\circ} \mathrm{C}$ with $5 \% \mathrm{CO}_{2}$ (this time point is set as time zero). After one hour of incubation, medium was removed and replaced by medium containing gentamycin $(50 \mu \mathrm{g} / \mathrm{mL})$ in order to kill extracellular bacteria, and then gentamycin $(10 \mu \mathrm{g} / \mathrm{mL})$. Note that for the experiments using IPTG, the IPTG $(10 \mathrm{mM})$ was kept during all the steps of the infection. At either $2 \mathrm{~h}, 4 \mathrm{~h}$ or $24 \mathrm{~h}$ post infection, cells were first washed with sterile PBS and were then incubated in PBS + Triton $0.1 \%$ at $37^{\circ} \mathrm{C}$ for $10 \mathrm{~min}$ in order to lyse the cells while keeping bacteria alive. After that, cells were flushed and lysates were harvested. Serial dilutions were performed and each dilution was spotted on $2 \mathrm{YT}$ agar plates and incubated at $37^{\circ} \mathrm{C}$.

\subsection{G1 Counting}

Bacteria in exponential phase of growth were diluted to an OD of 0.1 in 2YT liquid medium with or without IPTG. At each time point, $200 \mu \mathrm{L}$ of the culture was washed two times in PBS and bacteria were loaded onto a PBS agarose pad to be observed and counted by fluorescence microscopy.

Supplementary Materials: The following are available online at http://www.mdpi.com/2076-0817/9/7/571/s1, Figure S1: Effect of dksA deletion on macrophage infection; Figure S2: Codon-adapted mesh1b sequence; Figure S3: Fluorescence microscopy of the pSRK-relA' mCherry-parB strain induced with IPTG; Table S1: Number of bacteria counted in Figure 5.

Author Contributions: M.V.d.H. and X.D.B. designed the work and wrote the manuscript; M.V.d.H. and E.C. performed the experiments. All authors have read and agreed to the published version of the manuscript.

Funding: This work was funded by FRS-FNRS (PDR T.0060.15 and T.0058.20) and the University of Namur.

Acknowledgments: We thank Maxime Quebatte (Biozentrum, Basel) for the generous gift of the pMQ203 plasmid. We thank Severin Ronneau for the generous gift of the pSRK-relA' plasmid and for the numerous fruitful discussions on (p)ppGpp. M.V.d.H. was supported by a FRIA Ph.D. fellowship from FRS-FNRS. We thank the University of Namur for logistic support.

Conflicts of Interest: The authors declare no competing interests.

\section{References}

1. Moreno, E.; Moriyon, I. The Genus Brucella. Prokaryotes 2006, 5, 315-456.

2. Deghelt, M.; Mullier, C.; Sternon, J.F.; Francis, N.; Laloux, G.; Dotreppe, D.; Van der Henst, C.; Jacobs-Wagner, C.; Letesson, J.J.; De Bolle, X. The newborn Brucella abortus blocked at the G1 stage of its cell cycle is the major infectious bacterial subpopulation. Nat. Commun. 2014, 5, 4366. [CrossRef] [PubMed] 
3. Starr, T.; Ng, T.W.; Wehrly, T.D.; Knodler, L.A.; Celli, J. Brucella intracellular replication requires trafficking through the late endosomal/lysosomal compartment. Traffic 2008, 9, 678-694. [CrossRef] [PubMed]

4. Porte, F.; Liautard, J.P.; Kohler, S. Early acidification of phagosomes containing Brucella suis is essential for intracellular survival in murine macrophages. Infect. Immun. 1999, 67, 4041-4047. [CrossRef] [PubMed]

5. Pizarro-Cerda, J.; Meresse, S.; Parton, R.G.; van der Goot, G.; Sola-Landa, A.; Lopez-Goni, I.; Moreno, E.; Gorvel, J.P. Brucella abortus transits through the autophagic pathway and replicates in the endoplasmic reticulum of nonprofessional phagocytes. Infect. Immun. 1998, 66, 5711-5724. [CrossRef]

6. Starr, T.; Child, R.; Wehrly, T.D.; Hansen, B.; Hwang, S.; Lopez-Otin, C.; Virgin, H.W.; Celli, J. Selective subversion of autophagy complexes facilitates completion of the Brucella intracellular cycle. Cell Host Microbe 2012, 11, 33-45. [CrossRef] [PubMed]

7. Roop, R.M., 2nd; Gaines, J.M.; Anderson, E.S.; Caswell, C.C.; Martin, D.W. Survival of the fittest: How Brucella strains adapt to their intracellular niche in the host. Med. Microbiol. Immunol. 2009, 198, 221-238. [CrossRef]

8. Poncin, K.; Roba, A.; Jimmidi, R.; Potemberg, G.; Fioravanti, A.; Francis, N.; Willemart, K.; Zeippen, N.; Machelart, A.; Biondi, E.G.; et al. Occurrence and repair of alkylating stress in the intracellular pathogen Brucella abortus. Nat. Commun. 2019, 10, 4847. [CrossRef]

9. Kohler, S.; Foulongne, V.; Ouahrani-Bettache, S.; Bourg, G.; Teyssier, J.; Ramuz, M.; Liautard, J.P. The analysis of the intramacrophagic virulome of Brucella suis deciphers the environment encountered by the pathogen inside the macrophage host cell. Proc. Natl. Acad. Sci. USA 2002, 99, 15711-15716. [CrossRef]

10. Dozot, M.; Boigegrain, R.A.; Delrue, R.M.; Hallez, R.; Ouahrani-Bettache, S.; Danese, I.; Letesson, J.J.; De Bolle, X.; Kohler, S. The stringent response mediator Rsh is required for Brucella melitensis and Brucella suis virulence, and for expression of the type IV secretion system virB. Cell Microbiol. 2006, 8, 1791-1802. [CrossRef]

11. Kim, S.; Watanabe, K.; Suzuki, H.; Watarai, M. Roles of Brucella abortus SpoT in morphological differentiation and intramacrophagic replication. Microbiology 2005, 151, 1607-1617. [CrossRef] [PubMed]

12. Gonzalez, D.; Collier, J. Effects of (p)ppGpp on the progression of the cell cycle of Caulobacter crescentus. J. Bacteriol. 2014, 196, 2514-2525. [CrossRef] [PubMed]

13. Lesley, J.A.; Shapiro, L. SpoT regulates DnaA stability and initiation of DNA replication in carbon-starved Caulobacter crescentus. J. Bacteriol. 2008, 190, 6867-6880. [CrossRef] [PubMed]

14. Ronneau, S.; Petit, K.; De Bolle, X.; Hallez, R. Phosphotransferase-dependent accumulation of (p)ppGpp in response to glutamine deprivation in Caulobacter crescentus. Nat. Commun. 2016, 7, 11423. [CrossRef] [PubMed]

15. Schreiber, G.; Ron, E.Z.; Glaser, G. ppGpp-mediated regulation of DNA replication and cell division in Escherichia coli. Curr. Microbiol. 1995, 30, 27-32. [CrossRef]

16. Dalebroux, Z.D.; Edwards, R.L.; Swanson, M.S. SpoT governs Legionella pneumophila differentiation in host macrophages. Mol. Microbiol. 2009, 71, 640-658. [CrossRef]

17. Das, B.; Pal, R.R.; Bag, S.; Bhadra, R.K. Stringent response in Vibrio cholerae: Genetic analysis of spoT gene function and identification of a novel (p)ppGpp synthetase gene. Mol. Microbiol. 2009, 72, 380-398. [CrossRef]

18. Dahl, J.L.; Kraus, C.N.; Boshoff, H.I.; Doan, B.; Foley, K.; Avarbock, D.; Kaplan, G.; Mizrahi, V.; Rubin, H.; Barry, C.E., 3rd. The role of RelMtb-mediated adaptation to stationary phase in long-term persistence of Mycobacterium tuberculosis in mice. Proc. Natl. Acad. Sci. USA 2003, 100, 10026-10031. [CrossRef]

19. Paul, B.J.; Barker, M.M.; Ross, W.; Schneider, D.A.; Webb, C.; Foster, J.W.; Gourse, R.L. DksA: A critical component of the transcription initiation machinery that potentiates the regulation of rRNA promoters by ppGpp and the initiating NTP. Cell 2004, 118, 311-322. [CrossRef]

20. Ross, W.; Sanchez-Vazquez, P.; Chen, A.Y.; Lee, J.H.; Burgos, H.L.; Gourse, R.L. ppGpp Binding to a Site at the RNAP-DksA Interface Accounts for Its Dramatic Effects on Transcription Initiation during the Stringent Response. Mol. Cell 2016, 62, 811-823. [CrossRef]

21. Ronneau, S.; Hallez, R. Make and break the alarmone: Regulation of (p)ppGpp synthetase/hydrolase enzymes in bacteria. FEMS Microbiol. Rev. 2019, 43, 389-400. [CrossRef]

22. Mittenhuber, G. Comparative genomics and evolution of genes encoding bacterial (p)ppGpp synthetases/hydrolases (the Rel, RelA and SpoT proteins). J. Mol. Microbiol. Biotechnol. 2001, 3, 585-600. [PubMed] 
23. Plommet, M. Minimal requirements for growth of Brucella suis and other Brucella species. Zentralblatt Bakteriologie 1991, 275, 436-450. [CrossRef]

24. Ronneau, S.; Caballero-Montes, J.; Coppine, J.; Mayard, A.; Garcia-Pino, A.; Hallez, R. Regulation of (p)ppGpp hydrolysis by a conserved archetypal regulatory domain. Nucleic Acids Res. 2019, 47, 843-854. [CrossRef]

25. Sternon, J.F.; Godessart, P.; Goncalves de Freitas, R.; Van der Henst, M.; Poncin, K.; Francis, N.; Willemart, K.; Christen, M.; Christen, B.; Letesson, J.J.; et al. Transposon Sequencing of Brucella abortus Uncovers Essential Genes for Growth In Vitro and Inside Macrophages. Infect. Immun. 2018, 86. [CrossRef]

26. Sun, D.; Lee, G.; Lee, J.H.; Kim, H.Y.; Rhee, H.W.; Park, S.Y.; Kim, K.J.; Kim, Y.; Kim, B.Y.; Hong, J.I.; et al. A metazoan ortholog of SpoT hydrolyzes ppGpp and functions in starvation responses. Nat. Struct. Mol. Biol. 2010, 17, 1188-1194. [CrossRef]

27. Khan, S.R.; Gaines, J.; Roop, R.M., 2nd; Farrand, S.K. Broad-host-range expression vectors with tightly regulated promoters and their use to examine the influence of TraR and TraM expression on Ti plasmid quorum sensing. Appl. Environ. Microbiol. 2008, 74, 5053-5062. [CrossRef]

28. Hanna, N.; Ouahrani-Bettache, S.; Drake, K.L.; Adams, L.G.; Kohler, S.; Occhialini, A. Global Rsh-dependent transcription profile of Brucella suis during stringent response unravels adaptation to nutrient starvation and cross-talk with other stress responses. BMC Genom. 2013, 14, 459. [CrossRef]

29. Dozot, M.; Poncet, S.; Nicolas, C.; Copin, R.; Bouraoui, H.; Maze, A.; Deutscher, J.; De Bolle, X.; Letesson, J.J. Functional characterization of the incomplete phosphotransferase system (PTS) of the intracellular pathogen Brucella melitensis. PLoS ONE 2010, 5. [CrossRef]

30. Martinez-Nunez, C.; Altamirano-Silva, P.; Alvarado-Guillen, F.; Moreno, E.; Guzman-Verri, C.; Chaves-Olarte, E. The two-component system BvrR/BvrS regulates the expression of the type IV secretion system VirB in Brucella abortus. J. Bacteriol. 2010, 192, 5603-5608. [CrossRef]

31. Lacerda, T.L.; Salcedo, S.P.; Gorvel, J.P. Brucella T4SS: The VIP pass inside host cells. Curr. Opin. Microbiol. 2013, 16, 45-51. [CrossRef] [PubMed]

32. Terwagne, M.; Mirabella, A.; Lemaire, J.; Deschamps, C.; De Bolle, X.; Letesson, J.J. Quorum sensing and self-quorum quenching in the intracellular pathogen Brucella melitensis. PLoS ONE 2013, 8, e82514. [CrossRef] [PubMed]

33. Choi, K.H.; Gaynor, J.B.; White, K.G.; Lopez, C.; Bosio, C.M.; Karkhoff-Schweizer, R.R.; Schweizer, H.P. A Tn7-based broad-range bacterial cloning and expression system. Nat. Methods 2005, 2, 443-448. [CrossRef] [PubMed] 